\title{
EDITORIAL
}

\section{Fish stock assessments using surveys and indicators}

\author{
Guest Editors: Pierre Petitgas ${ }^{1}$, John Cotter $^{2}$, Verena Trenkel ${ }^{1}$ and Benoît Mesnil ${ }^{1}$
}

Conventional fish stock assessment methods are mostly dependent on commercial catch data. But there are more and more situations in which alternative assessment methods are needed. For instance, for stocks that are collapsed, the fishery might be closed so that fishery catches are unavailable or unreliable. Misreporting and unaccounted discards generate difficulties reliably converting landings to effective catches at sea. Also, advice is required for an increasing number of stocks, and for many of them historical series of fishery catches disaggregated by age do not exist, making conventional assessments impossible. In that context, research survey-based measurements made at sea with standardised methods and in known conditions represent an invaluable set of fishery-independent data on which to base an assessment. What type of assessment do these data lead to and how could such assessments be useful alongside or instead of existing methods?

The collection of papers in this special issue addresses these questions. The papers present methodology for single-species stock assessments and management strategies using only fishery-independent information from research surveys. Examples to illustrate the use of methods are also provided. For some papers scripts in the R language are attached on-line. The methods were developed within the EU project FISBOAT on Fishery Independent Survey Based Operational Assessment Tools (FP6 contract No. 502572; www.ifremer.fr/drvecohal/fisboat). They belong to three categories: monitoring procedures based on indicators of stock attributes, assessment models, and simulation evaluation tools. The two first categories of method are essentially diagnostic of stock changes. Simulation tools allow us to investigate the effects of management options and are complementary to the diagnostic tools.

The first paper presents some of the limitations of survey data (Trenkel and Cotter). The second paper examines some statistical issues that arise when estimating mean values of indicators from samples of fish collected by a trawl survey (Cotter). Subsequent papers are about indicator-based assessments. First, methods to compute population-level indices that are indicative of stock attributes are presented. These concern biological indicators (Cotter et al.) and also indicators of spatial distributions (Woillez et al.). Because many indicators are likely to be estimated, how to construct uncorrelated multivariate indicators is the subject of two papers (Petitgas and Poulard; Woillez et al.). Then, a set of methods to analyse indicator time series and detect temporal changes are presented in two papers. One (Cotter) reviews non parametric trend analysis methods. The other reviews statistical process control schemes, (e.g., cumulative sums: CUSUM) designed to monitor changes in the mean value along time series (Mesnil and Petitgas). To achieve an integrated assessment based on all the information from the analysis of the many indicator time series, an integration procedure is proposed (Petitgas) that uses CUSUM to construct a traffic light table of the deviations from a reference mean vector. The last paper in this group presents various survey-data-only assessment models (Mesnil et al.) and evaluates their performance using simulated data with different properties. All these survey-based methods lead to relative assessments based on testing for significant changes in many indicators time series. Because many indicators are used, the assessments have a broad biological basis.

Simulation tools are complementary to monitoring and assessment schemes as they allow testing of hypotheses and/or estimation of reference points or limits. They also allow testing of management options. This requires population models as well as harvest control rules. The length-based population simulation model ALADYM (Lembo et al.) is of interest in non-TAC based management contexts. Harvest control rules are reviewed by Apostolaki and Hillary and the many tools available in the simulation platform, FLR (Fisheries Library in R), are presented in the paper by Hillary. Finally, the paper by Cotter et al. summarises how comprehensive assessments might be carried out when using survey-based procedures either alongside conventional approaches, or instead of them as part of a drive towards a more broadly based, ecosystem approach to management of fisheries.

Many of the methods presented in this issue could serve for ecosystem monitoring and assessment as well as for fish stocks, one species at a time. They will hopefully contribute to the future development of fisheries and ecosystem assessments and, ultimately, to better, more sustainable management.

\footnotetext{
1 IFREMER, BP 21105, 44311 Cedex 3 Nantes, France

2 Fish World Science, The Avenue, Lowestoft, Suffolk, NR33 7LH, UK
} 